\title{
COVID-19 y comorbilidad en enfermería: a propósito de un caso
}

\author{
COVID-19 and comorbidity in nursing:in connection with a case
}

\author{
María Oyola-Canto « 1,a, Carmen Ricra-Echevarría 2,b, Yeni Girón-Mendoza 3,c, Vilma Yarasca-Quispe 4,d, \\ Hilda Torres-Figueroa ${ }^{5, e}$, Carlos Aldana-Contreras ${ }^{6, f}$
}

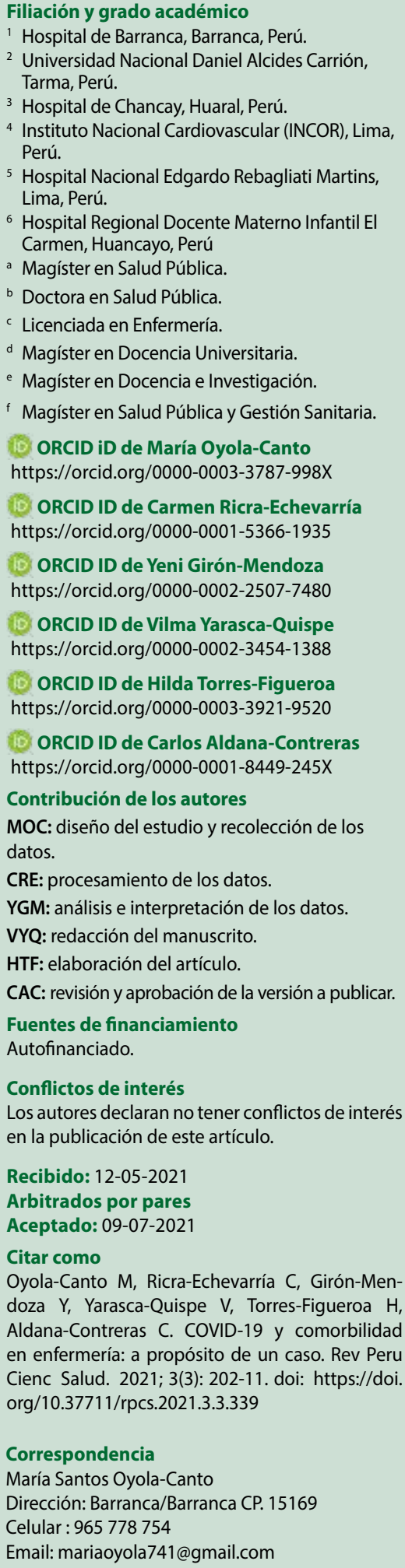

\section{RESUMEN}

Caso clínico sobre la aplicación del proceso enfermero en un paciente con comorbilidad (hipertensión arterial, obesidad y diabetes mellitus) y COVID-19, enfocado al modelo metodológico conceptual de Virginia Henderson vinculado al lenguaje estandarizado de la taxonomía NANDA, NOC y NIC. La finalidad del caso clínico es visibilizar el cuidado de enfermería, enfatizando la importancia de desarrollar un plan de cuidado enfocado en las respuestas humanas y las necesidades individuales del paciente, utilizando la metodología científica, generando conocimiento y cuidado de calidad. Su evidencia en un escenario clínico real nos permite poner de manifiesto la problemática que enfrenta el personal de enfermería en esta pandemia. Podemos concluir que el abordaje de los cuidados enfermeros en patologías complejas requiere de trabajo integrado y holístico, siendo necesario utilizar modelos metodológicos conceptuales para lograr los resultados deseados. El caso pretende estandarizar el lenguaje enfermero a través del plan de cuidado, proporcionando un marco de referencia para la práctica clínica.

Palabras clave: SARS-CoV-2; COVID-19; diabetes mellitus tipo 2; hipertensión arterial; obesidad (fuente: DeCS - BIREME).

\section{ABSTRACT}

Clinical case on the application of the nursing process in a patient with comorbidity (hypertension, obesity and diabetes mellitus) and COVID-19, focused on the conceptual methodological model of Virginia Henderson linked to the standardized language of the NANDA, NOC and NIC taxonomy. The purpose of the clinical case is to make nursing care visible, emphasizing the importance of developing a care plan focused on human responses and the individual needs of the patient, using scientific methodology, generating knowledge and quality care. Their evidence in a real clinical scenario allows us to highlight the problems faced by nursing staff in this pandemic. We can conclude that the approach of nursing care in complex pathologies requires integrated and holistic work, being necessary to use conceptual methodological models to achieve the desired results. The case aims to standardize nurse language through the care plan, providing a frame of reference for clinical practice.

Keywords: SARS-CoV2; COVID-19; diabetes mellitus type 2; hypertension; obesity (source: MeSH-NLM). 


\section{INTRODUCCIÓN}

El COVID-19 comenzó en Wuhan, China, a finales del 2019, diseminándose por todo el mundo (1). El primer caso en América se confirmó en Estados Unidos, el 20 de enero del 2020, y Brasil reportó el primer caso en América Latina y el Caribe en febrero del mismo año. Desde entonces, el COVID-19 se ha extendido a 54 países $\mathrm{y}$ regiones de las América ${ }^{(2)}$.

Al 26 de marzo del 2021 fueron reportados 125.174 .983 casos y 2.748.850 muertes por COVID-19 en el mundo (3). En Lima Metropolitana, al 15 de abril del 2021, se han confirmado 726712 casos y 22400 fallecidos por COVID-19 ${ }^{(4)}$. En la provincia de Barranca, el 16 de abril del 2021, fueron reportados 12349 casos y 554 fallecidos ${ }^{(5)}$.

Petrova ${ }^{\left({ }^{6}\right)}$ observó en su estudio que las enfermedades no transmisibles preexistentes empeoran el pronóstico de las infecciones por COVID-19, especialmente en pacientes con enfermedades cardiovasculares, respiratorias y metabólicas. Dentro de los factores que afectan la patogenia del virus se encuentran las comorbilidades de cada individuo como: obesidad, diabetes mellitus, hipertensión arterial y enfermedad cardiovascular, que podrían influir en el sistema inmune del huésped infectado por SARS-CoV-2.

Según la Organización Mundial de la Salud (OMS) la obesidad, es el índice de incremento de masa corporal (IMC) mayor o igual a $30 \mathrm{~kg} / \mathrm{m}^{2}$, se presenta en la mayoría de los casos de COVID-19(7).

El mecanismo fisiopatológico del COVID-19 se relaciona con procesos inflamatorios y de respuesta inmune humoral. El coronavirus ingresa a la célula a través de su proteína $\mathrm{S}$, utilizando como receptor a la angiotensina tipo II (ACE2), para multiplicar su carga viral y síntesis de citoquinas proinflamatorias. En pacientes con problemas respiratorios y obesidad existe mayor riesgo a complicaciones severas e ingreso a unidades críticas ${ }^{(8)}$.

La infección circunscrita al sistema respiratorio involucra a otros órganos, generando mayores complicaciones, falla multiorgánica, en consecuencia, un peor desenlace para el individuo. Las personas con obesidad tienen un alto riesgo de complicaciones graves de COVID-19 ${ }^{(8)}$.

Los pacientes con complicaciones por SARS-CoV-2 presentan insuficiencia respiratoria hipóxica en un $19 \%$; en China demostró que entre el $4 \%$ y el $13 \%$ de los pacientes recibieron ventilación con presión positiva no invasiva (VMNI) y entre el $2,3 \%$ y el $12 \%$ requirieron ventilación mecánica invasiva. Además, se reportó que, de 52 pacientes críticos, el $67 \%$ de estos pacientes desarrollaron síndrome de distrés respiratorio agudo; 33 de los cuales $(63,5 \%)$ recibieron cánula nasal de alto flujo (CNAF), haciendo parte regular del manejo del SDR ${ }^{(9)}$.

Las comorbilidades, que son factores de riesgo asociadas a complicaciones por COVID-19, son la hipertensión arterial (sobre todo si no es controlada), las enfermedades cardiovasculares graves, el cáncer y la diabetes mellitus ${ }^{(10)}$. Debido a la pandemia que está atravesando el mundo y nuestro país, se hace necesario tomar acciones decisivas y de impacto para beneficio del usuario interno y externo ante el coronavirus generado por el SARS-CoV-2 (COVID-19), lo que amerita generar la estandarización del cuidado de enfermería a pacientes con COVID-19(11). Bajo este contexto, el estudio toma como modelo del cuidado el marco filosófico de Virginia Henderson, de las 14 necesidades básicas: incluyendo desde los factores físicos hasta los psicológicos, vinculándolo además con las taxonomías NANDA, NOC y NIC, para conceptualizar, denominar, validar y clasificar los resultados que se obtienen de las respuestas humanas.

Virginia Henderson, en su modelo, buscó la independencia en la satisfacción de las necesidades fundamentales de la persona sana o enferma. Y para que esto sea posible se requiere que el profesional de enfermería posea conocimientos que le permitan incitar e incrementar las habilidades, destrezas y la voluntad del individuo hasta conseguir que sus requerimientos de salud puedan ser cubiertos de acuerdo a su capacidad ${ }^{(12)}$. Este modelo filosófico sirvió asimismo de guía de valoración para identificar las respuestas humanas, formular diagnósticos, planificar, ejecutar y evaluar las intervenciones.

El proceso de atención de enfermería es el método mediante el cual se aplica un amplio marco teórico a la práctica de enfermería. Utiliza un enfoque deliberativo en la resolución de problemas, que exige capacidades cognitivas, procedimentales e interpersonales en el profesional de enfermería; $y$, que tiene por objeto satisfacer las necesidades del paciente ${ }^{(13)}$. El conjunto de conocimientos y el desarrollo de habilidades y destrezas fortalecen además el actuar del profesional. Enfermería es ciencia, con un cuerpo teórico propio. El componente científico-técnico es fundamental para el ejercicio, sin embargo, el desarrollo del conocimiento y las habilidades blandas se complementan para un 
cuidado holístico y autónomo ${ }^{(14)}$. Durante el proceso de hospitalización la paciente recibe cuidado seguro, humano e integral, que se evidencia en la mejoría de algunos diagnósticos, y la presencia profesional del cuidado a través del acercamiento o abordaje del familiar a cabecera; sin embargo, el estado de salud de la paciente, por la misma evolución tórpida, condición clínica de comorbilidades y no disponibilidad oportuna de cama $\mathrm{UCl}$, se va deteriorando con falla multiorgánica, siendo trasladada a la UCl al séptimo día.

La práctica de valores y principios es fundamental en el actuar diario y la convivencia de todo ser humano; más aún en el profesional de enfermería quien brinda servicios de cuidados. La habilidad de interacción por un profesional de enfermería garantiza un cuidado humano y cálido. Virginia Henderson vuelca sus raíces a las bases fundamentales de la enfermería alejándose del enfoque biomédico y adoptando modelos de cuidados autónomos, siendo compatibles con el proceso de enfermería, herramienta fundamental y esencial para que tenga aplicación científica humanista en la práctica profesional ${ }^{(15)}$.

El proceso de atención de enfermería es un método dinámico y sistemático para brindar cuidado enfermero, promoviendo cuidados humanos que se centran en obtener resultados que motiven a los enfermeros a evaluarlos y plantearse cómo mejorarlos.

El lenguaje estandarizado de la enfermería anteriormente mencionado como NANDA, NOC y $\mathrm{NIC}$; es un instrumento que permite integrar un marco teórico de identificación de problemas, intervenciones y resultados en los cuidados de enfermería ${ }^{(16)}$.

El objetivo del presente estudio es, por lo tanto, brindar cuidado humano, oportuno y holístico individualizado a un paciente con comorbilidad preexistente y portador de COVID-19, en base a las respuestas humanas y a partir de la perspectiva filosófica de las 14 necesidades de Virginia Henderson, integrando las herramientas del lenguaje enfermero estandarizado NANDA, NOC y NIC.

\section{Presentación del caso}

Paciente mujer de 59 años de edad, obesa, hospitalizada en el área de emergencia COVID-19, despierta, con apoyo de máscara de reservorio. A la valoración se le encuentra lúcida, agitada y diaforética, la piel y mucosas se encuentran ligeramente húmedas y cianosis leve en zonas distales de manos y pies.
Se auscultan sibilancias y estertores, evidenciándose abundantes secreciones blanquecinas, con sonda nasogástrica para alimentación y catéter periférico sólo para tratamiento endovenoso. Portadora de sonda vesical, con una diuresis en 24 horas de 1600 cc, de características amarillo colúrica, siendo su gasto urinario $=0,73 \mathrm{cc} /$ hr, con apoyo de pañal. Luego de dos días, inicia una evolución tórpida con una saturación de oxígeno que oscila entre $85 \%$ a $90 \%$, frecuencia respiratoria a 32 por minuto, sensación de falta de aire, se coloca cánula de alto flujo a 32 litros con un $\mathrm{FIO}_{2} 50 \%$, manteniendo este soporte tres días sin mejoría alguna.

Al tercer día, presenta trastorno del sensorio, hipoactiva, con tendencia al sueño, en posición de pronación alcanza una saturación del $90 \%$, a ello se agrega la no tolerancia a la posición prona por mucho tiempo. Esperando pasar a la unidad de cuidados intensivos para manejo de ventilación invasiva con los siguientes diagnósticos agregados: insuficiencia respiratoria con ventilación no invasiva, síndrome de distrés respiratorio agudo, desequilibrio electrolítico.

\section{Antecedentes}

- Hipertensión arterial

- Diabetes mellitus tipo II

- Obesidad grado II

- Recibió vacunas: primera dosis (11 de marzo), segunda dosis (4 de abril), desarrolla síntomas (16 de marzo)

\section{Historia clínica}

Peso $91 \mathrm{~kg}$ y talla $1,55 \mathrm{~cm}$.

Diagnóstico: neumonía viral por SARS-CoV-2; síndrome de distrés respiratorio agudo, desequilibrio hidroelectrolítico.

Hemograma: leucocitos $11,870 \mathrm{~mm} 3$; plaquetas $228 \mathrm{~mm} 3$, sodio $149 \mathrm{mmol} / \mathrm{L}$; potasio $2,73 \mathrm{mmol} / \mathrm{L}$.

Perfil renal: urea $20 \mathrm{~mm} 3$, creatinina $0,9 \mathrm{~mm} 3$; glucosa: $132 \mathrm{mg} / \mathrm{dL}$.

Gasometría arterial: pH 7,49 PaCO2 38,1 mmHg, $\mathrm{PaO} 258 \mathrm{mmHg}, \mathrm{HCO} 329,2 \mathrm{mmol} / \mathrm{L}$.

Estado nutricional: $\mathrm{IMC}=37,87 \mathrm{Kg} / \mathrm{m}^{2}$. 
Antes de pasar a la unidad de cuidados intensivos: Funciones vitales: $T^{\circ} 38,1^{\circ} \mathrm{C}, \mathrm{FC}$ : 98 latidos por minuto; FR: 32 por minuto; $\mathrm{PA}: 110 / 70 \mathrm{mmHg}, \mathrm{SaO}_{2}: 78 \%$, diuresis 1600 cc en 24 horas.

\section{Valoración}

El modelo teórico de Virginia Henderson se basa en un modelo humanista, enfocándose en las 14 necesidades humanas, donde el rol principal del profesional de enfermería es cimentar la ciencia del cuidado, brindando cuidados de suplencia o ayuda al ser humano sano o enfermo en los diferentes ciclos de vida ${ }^{(17)}$.

\section{Las necesidades básicas alteradas son:}

\section{Respiración/circulación}

La paciente presenta una frecuencia respiratoria de 32 respiraciones por minuto, con una saturación de oxígeno que oscila entre $85 \%$ y $90 \%$. Al momento se toma AGA: $\mathrm{pH} 7,49 \mathrm{PaCO}_{2^{\prime}} 38,1 \mathrm{mmHg}, \mathrm{PaO}_{2} 58 \mathrm{mmHg}, \mathrm{HCO}_{3^{\prime}} 29,2$ $\mathrm{mmol} / \mathrm{L}$, pasando a cánula de alto flujo a 32 litros a un $\mathrm{FIO}_{2} 50 \%$. A la auscultación, sibilancias, estertores y abundantes secreciones blanquecinas, con antecedentes de hipertensión arterial.

Diagnóstico enfermero (00031): limpieza ineficaz de las vías aéreas relacionado con mucosidad excesiva, evidenciado por alteración en el patrón respiratorio y excesiva cantidad de esputo.

Diagnóstico enfermero (00030): deterioro del intercambio de gases relacionado con desequilibrio en la ventilación-perfusión, evidenciado por: $\mathrm{pH} 7,49$, $\mathrm{PaCO}_{2}, 38,1 \mathrm{mmHg}, \mathrm{PaO}_{2} 58 \mathrm{mmHg}, \mathrm{HCO}_{3^{\prime}} 29,2 \mathrm{mmol} / \mathrm{L}$.

\section{Alimentación/hidratación}

Su peso es de $91 \mathrm{~kg}$ y $1,55 \mathrm{~cm}$ de talla, con un IMC de $37,87 \mathrm{~kg} / \mathrm{m}^{2}$, presentando obesidad grado II, con sonda nasogástrica para alimentación. Antecedentes de diabetes mellitus tipo 2 , con mucosas orales secas y con un catéter periférico en miembro superior, sólo para tratamiento endovenoso.

Diagnóstico enfermero (00179): riesgo de nivel de glucemia inestable como se evidencia por control inadecuado de la glucemia.

\section{Eliminación}

Portadora de sonda vesical, con una diuresis en 24 horas de 1600 cc de características orina colúrica siendo su gasto urinario $=0,73 \mathrm{cc} / \mathrm{hr}$ con apoyo de pañal.

\section{Movilización}

Paciente en cama, con soporte de cánula de alto flujo, dispositivo de sujeción mecánica por inquietud y agitación, movilidad disminuida en posición prona no tolerando esta posición, agregado a ello su obesidad grado II.

Diagnóstico enfermero (00249): riesgo de úlcera por presión como se evidencia por largo periodo de inmovilidad y déficit de autocuidado.

\section{Termorregulación}

Paciente presenta temperatura axilar de $38,1^{\circ} \mathrm{C}$, piel caliente al tacto, mucosas orales secas.

Diagnóstico enfermero (0007): hipertermia relacionado con deshidratación evidenciado por piel caliente al tacto y temperatura de $38,1^{\circ} \mathrm{C}$.

\section{Higiene/piel}

Piel poco hidratada, ligera cianosis en zonas distales del cuerpo, baño de esponja.

\section{Comunicación}

Los primeros días de hospitalización lúcida, después de dos días con trastorno del sensorio y tendencia a la somnolencia.

Diagnóstico enfermero (00128): confusión aguda relacionada con deprivación sensorial evidenciado por agitación y somnolencia.

\section{DISCUSIÓN}

En el Perú, el Sistema Nacional de Defunciones (SINADEF), órgano del Ministerio de Salud (MINSA), informó que el $85,5 \%$ de los pacientes fallecidos por COVID-19 eran obesos, de modo que la obesidad incrementa el riesgo de muerte y las complicaciones en el paciente con COVID-19 ${ }^{(18)}$. En nuestro caso podemos identificar que la paciente tenía obesidad grado II que incrementa el riesgo de muerte, esto lo evidencia su IMC de $37,87 \mathrm{~kg} / \mathrm{m}^{2}$.

A nivel nacional, el 37,2 \% de las personas de 15 a más años de edad presentan comorbilidad: hipertensión arterial, diabetes mellitus $\mathrm{u}$ obesidad. Las personas de 80 a más años de edad presentan mayores porcentajes de comorbilidad (67,6\%). El porcentaje tiende a ser mayor en mujeres que en hombres ${ }^{(19)}$. En el estudio, la paciente es de sexo femenino y tiene 59 años, encontrándose dentro de los intervalos de factores de riesgo etario; 


\section{PLAN DE CUIDADOS DE ENFERMERÍA}

Tabla 1. Diagnóstico enfermero (00031): limpieza ineficaz de las vías aéreas relacionado con mucosidad excesiva evidenciado por alteración en el patrón respiratorio y excesiva cantidad de esputo ${ }^{(18)}$

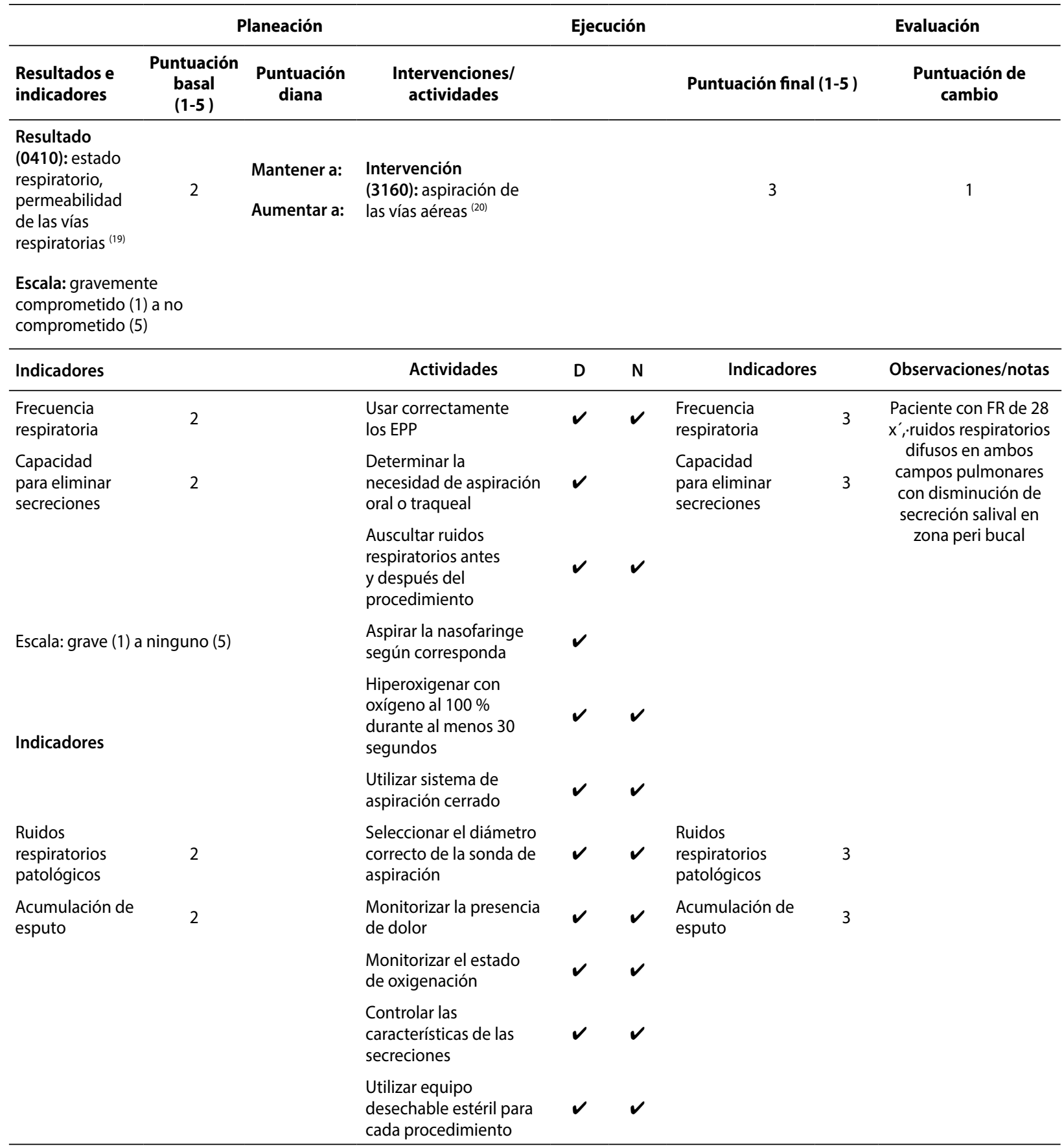

así mismo, se reportó en la paciente antecedentes de hipertensión arterial, diabetes mellitus tipo Il y obesidad.

Giral Herrera ${ }^{(20)}$ estudió la relación existente entre COVID-19 e hipertensión buscando identificar la relación existente entre COVID-19 e hipertensión; allí realiza revisión bibliográfica y de literatura de cardiología, OMS/OPS y sociedades científicas, observando una clara relación entre el antecedente de hipertensión arterial y el curso evolutivo desfavorable en pacientes COVID-19, determinando que la enzima conversora de angiotensina actúa como receptor del SARS-Cov- 2 y los pacientes con hipertensión arterial presentan un curso menos favorable en el COVID-19. La paciente inicia sintomatología: malestar general, dolor muscular y cefalea intensa, se realiza la prueba antigénica saliendo positiva al COVID-19, por lo que 
Tabla 2. Diagnóstico enfermero (00030): deterioro del intercambio de gases relacionado con desequilibrio en la ventilación-perfusión evidenciado: $\mathrm{pH} 7,49 \mathrm{PaCO}_{2}, 38,1 \mathrm{mmHg}, \mathrm{PaO}_{2} 58 \mathrm{mmHg}, \mathrm{HCO}_{3}, 29,2 \mathrm{mmol} / \mathrm{L}^{(18)}$

\begin{tabular}{|c|c|c|c|c|c|c|c|c|}
\hline \multicolumn{4}{|c|}{ Planeación } & \multicolumn{4}{|c|}{ Ejecución } & \multirow{2}{*}{$\begin{array}{c}\text { Evaluación } \\
\begin{array}{c}\text { Puntuación de } \\
\text { cambio }\end{array}\end{array}$} \\
\hline $\begin{array}{l}\text { Resultados e } \\
\text { Indicadores }\end{array}$ & $\begin{array}{l}\text { Puntuación } \\
\text { basal (1-5) }\end{array}$ & $\begin{array}{l}\text { Puntuación } \\
\text { diana }\end{array}$ & Intervenciones/actividades & & & $\begin{array}{c}\text { Puntuación fir } \\
\qquad(1-5)\end{array}$ & & \\
\hline $\begin{array}{l}\text { Resultado } \\
\text { (0402): estado } \\
\text { respiratorio: } \\
\text { intercambio de } \\
\text { gases }^{(19)}\end{array}$ & 2 & $\begin{array}{l}\text { Mantener a: } \\
\text { Aumentar a: }\end{array}$ & $\begin{array}{l}\text { Intervención } \\
\text { (3320): oxigenoterapia }{ }^{(20)}\end{array}$ & & & 2 & & 0 \\
\hline \multicolumn{9}{|c|}{$\begin{array}{l}\text { Escala: desviación grave } \\
\text { del rango normal (1) a sin } \\
\text { desviación del rango normal (5) }\end{array}$} \\
\hline & Indicadores & & Actividades & D & $\mathrm{N}$ & Indicadores & & $\begin{array}{c}\text { Observaciones/ } \\
\text { notas }\end{array}$ \\
\hline $\mathrm{PaO}_{2}$ & 2 & & $\begin{array}{l}\text { Mantener la permeabilidad de las } \\
\text { vías aéreas. }\end{array}$ & $\checkmark$ & $\checkmark$ & $\mathrm{PaO}_{2}$ & 2 & \multirow{9}{*}{$\begin{array}{l}\text { Paciente con } \\
\text { pH inestable } \\
\text { con saturación } \\
\text { de oxigeno } \\
\text { por cánula } \\
\text { de alto flujo a } \\
\text { con fio de } 32 \\
\text { con tendencia } \\
\text { al sueño a } \\
\text { intervalos }\end{array}$} \\
\hline $\mathrm{PaCO}_{2}$ & 4 & & $\begin{array}{l}\text { Administrar oxígeno suplementario } \\
\text { según indicación }\end{array}$ & $\boldsymbol{v}$ & $\checkmark$ & $\mathrm{PaCO}_{2}$ & 4 & \\
\hline Saturación de $\mathrm{O}_{2}$ & 2 & & Vigilar el flujo de litros de oxígeno & $v$ & $\checkmark$ & Saturación de $\mathrm{O}_{2}$ & 2 & \\
\hline Escala: grave (1) & a ninguno (5) & & $\begin{array}{l}\text { Comprobar la posición correcto del } \\
\text { dispositivo de aporte de oxígeno }\end{array}$ & $\checkmark$ & $\checkmark$ & & & \\
\hline \multirow{2}{*}{ Indicadores } & & & $\begin{array}{l}\text { Vigilar la piel en busca de fricción } \\
\text { por el dispositivo de oxígeno }\end{array}$ & $\checkmark$ & $\checkmark$ & & & \\
\hline & & & $\begin{array}{l}\text { Observar signos de hipoventilación } \\
\text { inducida por el oxígeno }\end{array}$ & $\checkmark$ & $\checkmark$ & & & \\
\hline \multirow[t]{3}{*}{ Somnolencia } & 2 & & $\begin{array}{l}\text { Observar la ansiedad del paciente } \\
\text { relacionado con la necesidad de } \\
\text { oxígeno }\end{array}$ & $\checkmark$ & $\checkmark$ & Somnolencia & 2 & \\
\hline & & & $\begin{array}{l}\text { Valorar e interpretar los valores de } \\
\text { gasometría arterial }\end{array}$ & & & & & \\
\hline & & & $\begin{array}{l}\text { Comprobar la eficacia de la } \\
\text { oxigenoterapia a través del } \\
\text { pulsioxímetro y gasometría arterial }\end{array}$ & $\checkmark$ & $\checkmark$ & & & \\
\hline
\end{tabular}

inicia aislamiento domiciliario. Al tercer día es ingresada al servicio de emergencia por desaturación, dificultad respiratoria, cansancio al caminar y malestar general, con curso evolutivo desfavorable. También, según el Centro Nacional de Epidemiología, Prevención y Control de Enfermedades, del Ministerio de Salud, confirman que tanto la obesidad como la diabetes mellitus son dos condiciones que se encuentran muy relacionadas a la severidad del cuadro clínico y al riesgo de morir en paciente portador de COVID-19 ${ }^{(21)}$.

Estudios realizados en Reino Unido, en mayo del 2020, en pacientes críticamente enfermos con COVID-19 confirmados, se halló, en una cohorte con 3508 pacientes con necesidad de soporte vital avanzado que 1273 cumplían con un IMC $\geq 30^{(22)}$ y, en New York, en un estudio de serie de casos, con 5700 pacientes positivos a COVID-19, realizado por Richardson et al. ${ }^{(23)}$, concluyó, que las comorbilidades más frecuentes en este grupo de pacientes fueron obesidad, hipertensión y diabetes. Simonnet et al. ${ }^{(24)}$, en un estudio retrospectivo de cohorte, en 124 pacientes con COVID-19, evidenció que la incidencia de necesidad de ventilación mecánica invasiva fue mayor en pacientes con IMC $\geq 30$ y con mayor impacto en pacientes con IMC $\geq 35 \mathrm{~kg} / \mathrm{m}^{2}$. En el hospital de la Universidad de Lyon (Francia); Causy y et al. ${ }^{(25)}$, en un estudio de cohorte, en 291 pacientes con SARS-CoV-2, 124 pacientes prolongaron su estancia hospitalaria, con necesidades de oxígeno suplementario con sistemas de bajo flujo y un tercio de esta muestra necesitó ventilación invasiva temprana y tenían un $I M C \geq 35 \mathrm{~kg} / \mathrm{m}^{2}$, concluyendo que la obesidad es un factor de gravedad para COVID-19.

Cabe señalar que en un estudio descriptivo, transversal, prospectivo, con 73 profesionales de enfermería, el $54,8 \%$ presentó sobrepeso y el $17,8 \%$ obesidad. La alta prevalencia de sobrepeso sumado al sedentarismo y el perfil lipídico incrementado fue predominante en esta población adulta ${ }^{(26)}$. Otro estudio realizado en Asunción concluyó que el estilo de vida en los profesionales de la 
Tabla 4. Diagnóstico enfermero (00128): confusión aguda relacionada con deprivación sensorial evidenciado por agitación y alteración del funcionamiento cognitivo ${ }^{(18)}$

\begin{tabular}{|c|c|c|c|c|c|c|c|c|}
\hline & \multicolumn{3}{|c|}{ Planeación } & \multicolumn{4}{|c|}{ Ejecución } & Evaluación \\
\hline $\begin{array}{l}\text { Resultados e } \\
\text { indicadores }\end{array}$ & $\begin{array}{l}\text { Puntuación } \\
\text { basal (1-5) }\end{array}$ & $\begin{array}{l}\text { Puntuación } \\
\text { diana }\end{array}$ & Intervenciones/actividades & & & $\begin{array}{l}\text { Puntuación final } \\
\qquad(1-5)\end{array}$ & & $\begin{array}{l}\text { Puntuación } \\
\text { de cambio }\end{array}$ \\
\hline $\begin{array}{l}\text { Resultado } \\
\text { (0912): estado } \\
\text { neurológico, } \\
\text { consciencia (19) }\end{array}$ & 3 & $\begin{array}{l}\text { Mantener a: } \\
\text { Aumentar a: }\end{array}$ & $\begin{array}{l}\text { Intervención (2620): monitorización neurológica } \\
(20)\end{array}$ & & & 4 & & 1 \\
\hline \multicolumn{9}{|c|}{$\begin{array}{l}\text { Escala: gravemente comprometido } \\
\text { (1) a no comprometido (5) }\end{array}$} \\
\hline Indicadores & & & Actividades & D & $\mathrm{N}$ & Indicadores & & $\begin{array}{l}\text { Observacio- } \\
\text { nes/notas }\end{array}$ \\
\hline $\begin{array}{l}\text { Abre los ojos a estí- } \\
\text { mulos externos }\end{array}$ & 3 & & Vigilar el nivel de conciencia & $\boldsymbol{v}$ & $\checkmark$ & $\begin{array}{l}\text { Abre los ojos a estímu- } \\
\text { los externos }\end{array}$ & 4 & $\begin{array}{l}\text { El estado de } \\
\text { conciencia se }\end{array}$ \\
\hline Orientación cognitiva & 3 & & Vigilar las tendencias en la escala del coma de Glasgow & $\checkmark$ & $\boldsymbol{v}$ & Orientación cognitiva & 4 & $\begin{array}{c}\text { encuentra con } \\
\text { tendencia al }\end{array}$ \\
\hline $\begin{array}{l}\text { Comunicación apro- } \\
\text { piada a la situación }\end{array}$ & 4 & & $\begin{array}{l}\text { Analizar la memoria reciente, nivel de atención, } \\
\text { memoria pasada, humor, afectos y conductas }\end{array}$ & $\checkmark$ & $\boldsymbol{v}$ & $\begin{array}{l}\text { Comunicación } \\
\text { apropiada a la } \\
\text { situación }\end{array}$ & 4 & $\begin{array}{l}\text { sueño; pero } \\
\text { a estímulos } \\
\text { fuertes }\end{array}$ \\
\hline \multicolumn{2}{|c|}{ Escala: grave (1) a ninguno (5) } & & $\begin{array}{l}\text { Comprobar el estado respiratorio, niveles de } \\
\text { gases arteriales, pulsoximetria, profundidad de la } \\
\text { frecuencia respiratoria }\end{array}$ & $\checkmark$ & $\boldsymbol{v}$ & & & $\begin{array}{l}\text { responde. } \\
\text { Con coma de } \\
\text { Glasgow: } 12 \\
\text { puntos }\end{array}$ \\
\hline & & & Vigilar las características del habla & $\boldsymbol{v}$ & $\boldsymbol{v}$ & & & \\
\hline Indicadores & & & $\begin{array}{l}\text { Comprobar la respuesta a los estímulos verbal, táctil } \\
\text { y nocivas }\end{array}$ & $\boldsymbol{v}$ & $\checkmark$ & & & \\
\hline Estupor & 5 & & & $\checkmark$ & $\nu$ & Estupor & 5 & \\
\hline
\end{tabular}

salud fue predominante poco saludable, detectándose un estado nutricional con valores superiores a la normalidad, según el IMC ${ }^{(27)}$.
Fernández et al. ${ }^{(28)}$ investigaron el juicio de las enfermeras acerca de un plan de cuidados estandarizado de infección por coronavirus (COVID-19), cuyo objetivo

Tabla 5. Diagnóstico enfermero (00179): riesgo de nivel de glucemia inestable como se evidencia por control inadecuado de la glucemia ${ }^{(18)}$

\begin{tabular}{|c|c|c|c|c|c|c|c|c|}
\hline \multicolumn{4}{|c|}{ Planeación } & \multicolumn{4}{|c|}{ Ejecución } & \multirow{2}{*}{$\begin{array}{c}\text { Evaluación } \\
\begin{array}{c}\text { Puntuación de } \\
\text { cambio }\end{array}\end{array}$} \\
\hline $\begin{array}{l}\text { Resultados e } \\
\text { indicadores }\end{array}$ & $\begin{array}{l}\text { Puntuación } \\
\text { basal (1-5) }\end{array}$ & $\begin{array}{l}\text { Puntuación } \\
\text { diana }\end{array}$ & Intervenciones/actividades & & & $\begin{array}{c}\text { Puntuación fin } \\
(1-5)\end{array}$ & & \\
\hline $\begin{array}{l}\text { Resultado } \\
\text { (2300): nivel de } \\
\text { glucemia }^{(19)}\end{array}$ & 5 & $\begin{array}{l}\text { Mantener a: } \\
\text { Aumentar a: }\end{array}$ & $\begin{array}{l}\text { Intervención } \\
\text { (2120): manejo de hiperglucemia }{ }^{(20)}\end{array}$ & & & 5 & & 0 \\
\hline \multicolumn{9}{|c|}{$\begin{array}{l}\text { Escala: gravemente comprometido } \\
\text { (1) a no comprometido (5) }\end{array}$} \\
\hline Indicadores & & & Actividades & D & $\mathrm{N}$ & Indicadores & & $\begin{array}{c}\text { Observaciones/ } \\
\text { notas }\end{array}$ \\
\hline $\begin{array}{l}\text { Concentración } \\
\text { sanguínea de glucosa }\end{array}$ & 3 & & Vigilar los niveles de glucosa en sangre & $\checkmark$ & $\checkmark$ & $\begin{array}{l}\text { Concentración } \\
\text { sanguínea de } \\
\text { glucosa }\end{array}$ & 4 & $\begin{array}{l}\text { Paciente presenta } \\
\text { en los días } \\
\text { consecutivos una }\end{array}$ \\
\hline $\begin{array}{l}\text { Hemoglobina } \\
\text { glicosilada }\end{array}$ & 5 & & $\begin{array}{l}\text { Observar los signos y síntomas de } \\
\text { hiperglucemia, poliuria, polidipsia, polifagia, } \\
\text { debilidad, malestar, letargia }\end{array}$ & $\checkmark$ & $\checkmark$ & $\begin{array}{l}\text { Hemoglobina } \\
\text { glicosilada }\end{array}$ & 5 & $\begin{array}{l}\text { glicemia de } 121 \\
\text { g/dL }\end{array}$ \\
\hline Glucosa en orina & 5 & & $\begin{array}{l}\text { Vigilar la presencia de cuerpos cetónicos } \\
\text { en orina. }\end{array}$ & $\checkmark$ & $\checkmark$ & Glucosa en orina & 5 & \\
\hline \multirow[t]{4}{*}{ Cetonas en orina } & 5 & & $\begin{array}{l}\text { Anticiparse en aquellas situaciones en } \\
\text { las que aumentaran las necesidades de } \\
\text { insulina }\end{array}$ & $\checkmark$ & $\checkmark$ & Cetonas en orina & 5 & \\
\hline & & & Realizar el balance hídrico & $\boldsymbol{V}$ & $\checkmark$ & & & \\
\hline & & & Valorar el estado neurológico & $\boldsymbol{V}$ & & & & \\
\hline & & & Monitorizar la presión arterial y pulso & $\boldsymbol{V}$ & $\checkmark$ & & & \\
\hline
\end{tabular}


Tabla 6. Diagnostico enfermero (00249): riesgo de úlcera por presión como se evidencia por largo periodo de inmovilidad y déficit de autocuidado ${ }^{(18)}$

\begin{tabular}{|c|c|c|c|c|c|c|c|c|}
\hline & \multicolumn{3}{|c|}{ Planeación } & \multicolumn{4}{|c|}{ Ejecución } & \multirow{2}{*}{$\begin{array}{c}\text { Evaluación } \\
\begin{array}{c}\text { Puntuación de } \\
\text { cambio }\end{array}\end{array}$} \\
\hline $\begin{array}{l}\text { Resultados e } \\
\text { indicadores }\end{array}$ & $\begin{array}{l}\text { Puntuación } \\
\text { basal (1-5) }\end{array}$ & $\begin{array}{l}\text { Puntuación } \\
\text { diana }\end{array}$ & Intervenciones/actividades & & & $\begin{array}{c}\text { Puntuación fir } \\
(1-5)\end{array}$ & & \\
\hline $\begin{array}{l}\text { Resultado } \\
\text { (1101): integridad } \\
\text { tisular: piel } \\
\text { y membranas } \\
\text { mucosas }{ }^{(19)}\end{array}$ & 5 & $\begin{array}{l}\text { Mantener a: } \\
\text { Aumentar a: }\end{array}$ & $\begin{array}{l}\text { Intervención } \\
\text { (3540): prevención de úlceras por } \\
\text { presión }{ }^{(20)}\end{array}$ & & & 5 & & 0 \\
\hline \multicolumn{9}{|c|}{$\begin{array}{l}\text { Escala: gravemente comprometido } \\
\text { (1) a no comprometido (5) }\end{array}$} \\
\hline Indicadores & & & Actividades & D & $\mathrm{N}$ & Indicadores & & $\begin{array}{l}\text { Observaciones/ } \\
\text { notas }\end{array}$ \\
\hline Sensibilidad & 5 & & $\begin{array}{l}\text { Valorar el riesgo a través de la escala de } \\
\text { Braden }\end{array}$ & $\boldsymbol{v}$ & $\boldsymbol{v}$ & Sensibilidad & 5 & $\begin{array}{l}\text { La integridad } \\
\text { tisular de la }\end{array}$ \\
\hline Perfusión tisular & 4 & & Documentar el peso y cambio de peso & $\checkmark$ & $\checkmark$ & Perfusión tisular & 5 & $\begin{array}{l}\text { paciente se } \\
\text { mantiene piel }\end{array}$ \\
\hline $\begin{array}{l}\text { Integridad de } \\
\text { la piel }\end{array}$ & 5 & & Registrar el estado de la piel por turno & $\boldsymbol{v}$ & $\checkmark$ & $\begin{array}{l}\text { Integridad de } \\
\text { la piel }\end{array}$ & 5 & $\begin{array}{l}\text { integra sin } \\
\text { lesiones }\end{array}$ \\
\hline \multirow{2}{*}{\multicolumn{2}{|c|}{ Escala: grave (1) a ninguno (5) }} & & $\begin{array}{l}\text { Vigilar estrechamente cualquier zona } \\
\text { enrojecida }\end{array}$ & $\boldsymbol{v}$ & $v$ & & & \\
\hline & & & Eliminar la humedad excesiva en la piel & $\boldsymbol{v}$ & $\boldsymbol{v}$ & & & \\
\hline Indicadores & & & $\begin{array}{l}\text { Aplicar barreras de protección como } \\
\text { cremas hidratantes }\end{array}$ & $\boldsymbol{v}$ & $\checkmark$ & & & \\
\hline Eritema & 5 & & $\begin{array}{l}\text { Cambiar la posición cada } 2 \text { horas según } \\
\text { corresponda }\end{array}$ & $\boldsymbol{v}$ & $\checkmark$ & Eritema & 5 & \\
\hline \multirow[t]{7}{*}{ Palidez } & 5 & & $\begin{array}{l}\text { Colocar el programa de cambios postu- } \\
\text { rales en la cama }\end{array}$ & $\boldsymbol{v}$ & $v$ & Palidez & 5 & \\
\hline & & & $\begin{array}{l}\text { Inspeccionar la piel en las prominencias } \\
\text { óseas }\end{array}$ & $\boldsymbol{v}$ & $\checkmark$ & & & \\
\hline & & & $\begin{array}{l}\text { Elevar los puntos de presión con } \\
\text { almohadas }\end{array}$ & $\boldsymbol{v}$ & $\checkmark$ & & & \\
\hline & & & $\begin{array}{l}\text { Evitar dar masajes en los puntos de } \\
\text { presión enrojecidas }\end{array}$ & $\boldsymbol{v}$ & $\checkmark$ & & & \\
\hline & & & Utilizar camas y colchones especiales & $\checkmark$ & $\checkmark$ & & & \\
\hline & & & Humedecer la piel seca intacta & $\boldsymbol{v}$ & $\checkmark$ & & & \\
\hline & & & $\begin{array}{l}\text { Aplicar protectores para los codos y los } \\
\text { talones }\end{array}$ & $\boldsymbol{v}$ & $\checkmark$ & & & \\
\hline
\end{tabular}

fue evaluar el contenido y diseño del plan de cuidados estandarizado de infección por COVID-19; donde se concluyó que existen altos índices de efectividad del planteamiento y diseño del plan de cuidados como herramienta para aplicar en la práctica clínica. Así mismo, en la presente investigación se evidenció la labor del profesional de enfermería usando el proceso de atención de enfermería (PAE), herramienta que garantiza la continuidad de los cuidados, potenció el rol autónomo de la enfermera y mejora la calidad de atención y seguridad del paciente.

La aplicación del PAE como método científico en la práctica asistencial, permite al profesional de enfermería prestar sus cuidados de manera racional, lógica, sistemática y holística; vinculando la taxonomía NANDA, NOC y NIC, al representar el conocimiento de la ciencia de enfermería.

El plan de cuidado estandarizado es un protocolo de actuación de enfermería basado en las necesidades de cuidado que presenta el paciente; una vez implementado y monitoreado consolida la evaluación de la intervención como eje del cuidado ${ }^{(29,30)}$.

El reporte de caso de la paciente de 59 años de edad, con diferentes comorbilidades como: hipertensión, obesidad, diabetes mellitus, contribuyeron a una evolución con pronóstico negativo, como también reporta Subham et al. ${ }^{(31)}$, para quienes la gravedad de la enfermedad COVID-19 se intensifica en pacientes con niveles elevados de glucosa probablemente a través 
de respuesta de citocina proinflamatoria amplificada, inmunidad innata deficiente y enzima convertidora de angiotensina 2 (ECA) regulada a la baja. Por tanto, el uso de inhibidores de la ECA o bloqueadores del receptor de angiotensina podría empeorar el nivel de glucosa en pacientes que padecen una infección por el nuevo coronavirus. También observaron que el daño directo de las células $\beta$ causado por el virus, la hipopotasemia y el aumento de la resistencia a la insulina mediado por citocinas y fetuina-A podrían deteriorar, asimismo, la condición diabética en pacientes con COVID-19. La evolución clínica de la patología con diabetes mellitus con un valor de glucosa de $132 \mathrm{mg} / \mathrm{dL}$, tienen similitud, ya que en el reporte de caso se evidenció la respuesta de cascada citocina-pro inflamatoria, deshidratación, somnolencia, datos que respaldan el reporte de Subham et al. Asimismo, el tratamiento reportado fue realizado con el mismo objetivo de mantener a los pacientes bien hidratados y seguir las recomendaciones para prevenir infecciones.

\section{Conclusiones}

El presente caso clínico es la aplicación del proceso enfermero, utilizando la valoración del modelo teórico de 14 necesidades básicas de Virginia Henderson que genera conocimiento y diagnósticos precisos en la paciente afectada con coronavirus SARS-CoV-2, asociado a comorbilidad de hipertensión arterial, diabetes mellitus Il y obesidad.

El caso clínico será un referente para facilitar al alumno o profesional de enfermería la vinculación teórico-metodológica de taxonomías NANDA, NOC y NIC en un escenario clínico real; así como para desarrollar habilidades y destrezas en la práctica clínica, unificar criterios de valoración desde un enfoque holístico y filosófico de Virginia Henderson, haciendo camino hacia la estandarización del lenguaje enfermero. Con este estudio se pretende estandarizar el cuidado de enfermería a través del plan de cuidados, proporcionando un marco de referencia para la práctica clínica.

\section{REFERENCIAS}

1. Acosta G, Escobar G, Bernaola G, Alfaro J, Taype Waldo, Marcos C, Amado J. Caracterización de pacientes con COVID-19 grave atendidos en un hospital de referencia nacional del Perú. Revista Perú Médica Exp. Salud Publica. 2020; 2(37): 259-8.

2. Organización Panamericana de la Salud (OPS). Respuesta de la organización panamericana de la salud a la COVID-19 en la región de las Américas [Internet] [Consultado $2021 \mathrm{Abr}$ 15] Disponible en: https://iris. paho.org/bitstream/handle/10665.2/52454/paho-res- ponse-covid-19-americas_spa.pdf?sequence $=5$ \&isAllowed=y

3. Luzuriaga J, Marsico F, García E, González V, Kreplak N, González S, et al. Impacto de la aplicación de vacunas contra COVID-19 sobre la incidencia de nuevas infecciones por SARS-COV-2 en PS de la Provincia de Buenos Aires. SciELO Preprints. En prensa. 2021.

4. Ministerio de Salud (MINSA). Situación Actual COVID-19. Perú; 2020-2021. Lima: MINSA; 2021.

5. Dirección Regional de Salud de Lima (DIRESA Lima). La sala contingencial COVID-19. Lima: DIRESA; 2020.

6. Petrova $D$, Salamanca $E$, Rodríguez $M$, Navarro $P$, Jiménez J, Sánchez M. La obesidad como factor de riesgo en personas con COVID-19: posibles mecanismos e implicaciones. Atención primaria [Internet]. 2020 [Consultado 2021 Abr 18]; 52(7): 496-500. Disponible en: https:// doi.org/10.1016/j.aprim.2020.05.003

7. Morales L, Ruvalcaba J. La obesidad, un verdadero problema de salud pública persistente en México. Journal of Negative and No Positive Results: JONNPR [Internet]. 2018 [Consultado 2021 Abr 20]; 3(8): 643-54. Disponible en: https://dialnet.unirioja.es/servlet/articulo?codigo $=6521709$

8. Tenorio J, Hurtado Y. Revisión sobre obesidad como factor de riesgo para mortalidad por COVID-19. Acta Médica Perú [Internet]. 2020 [Consultado 2021 Abril 14]; 37(3): 324-9. Disponible en: http://dx.doi.org/10.35663/ amp.2020.373.1197

9. Wang K, Zhao W, Li J, Shu W, Duan J. The experience of high-flow nasal cannula in hospitalized patients with 2019 novel coronavirus-infected pneumonia in two hospitals of Chongqing, China. Ann Intensive Care. 2020; 10(1): 0-4.

10. COVID-19 y comorbilidades - AMÉRICAS [Internet] Washington D. C.: Organización Panamericana de la Salud (OPS); 2020 [Consultado 2021 Abril 15] Disponible en: https://docs.bvsalud.org/biblioref/2020/07/1104403/ faq-underlying-conditions-tool-covid-19-spa.pdf

11. Chen G, Wu D, Guo W, Cao Y, Huang D, Wang H. Evidencia sobre el tratamiento de Covid-19. J Clin Invest. 2020;130(5): 192.

12. García D, Alba A, Cárdenas V. Proceso enfermero desde el modelo de cuidados de Virgina Henderson y los lenguajes NNN [Internet] [Consultado 2021 May 21] Disponible en: http://www.index-f.com/lascasas/documentos/lc0714.pdf

13. Fernández C, Torres M y Ruiz E. Teoría y práctica de los fundamentos de enfermería (I) [Internet] [Consultado 2021 May 21] Disponible en: https://www.researchgate. net/publication/345894569_Teoria_y_practica_de_los_ fundamentos_de_enfermeria_I_Bases_teoricas_y_metodologicas

14. Rodríguez P. y Báez F. Epistemología de la profesión enfermera. Revista Ene Revista de Enfermería [Internet]. 2020 [Consultado 2021 May 21]; 14(2). Disponible en: http://www.ene-enfermeria.org/ojs/index.php/ENE/article/view/958

15. Martínez RR. Modelos de enfermería integrados al cuidado del paciente nefrológico en el contexto cubano. Rev. Cubana Enferm. 2012; 28(4): 474-84.

16. Cachón Pérez JM, Álvarez-López C, Palacios - Ceña D. El significado del lenguaje estandarizado NANDA-NICNOC en las enfermeras de cuidados intensivos madrileñas: Abordaje fenomenológico. Enferm. Intensiva. 2012; 23(2): 68-76. 
17. Fernández $C$, Torres $M$, y Navarro E. Teoría y práctica de los fundamentos de enfermería (I). España: Editor Almeira; 2020.

18. Fiestas F, Peralta V, Zavala J, Barrionuevo P. Instituto de Evaluación de Tecnologías en Salud e Investigación Reporte Breve No 36 Obesidad como Factor de Riesgo. letsi - Essalud [Internet] [Consultado 2021 Abr 28] Disponible en: http://www.essalud.gob.pe/ietsi/pdfs/covid_19/ RB_36_Obesidad_30_jul_20.pdf

19. Instituto Nacional de Estadística e Informática (INEI). PERÚ: Factores de Riesgo Asociados a Complicaciones por COVID-19 ENDES 2018-2019. Lima: INEl; 2020.

20. Giralt-Herrera A, Rojas-Velázquez JM, Leiva-Enríquez J. Relación entre COVID-19 e Hipertensión Arterial. Rev habanera cienc méd. 2020; 19(2):1-11.

21. Ministerio de Salud (MINSA). Semana Epidemiológica 53.29 Boletín Epidemiológico del Perú 2020 [Internet] [Consultado 2021 May 21] Disponible en: https:// www.dge.gob.pe/epipublic/uploads/boletin/boletin_202053.pdf

22. Docherty A, Harrison E, Verde C, Hardwick H, Pius R, Norman L, Holden K, Leer J, Dondelinger F, Carson G, Merson $\mathrm{L}$, et al. Características de 16.749 pacientes hospitalizados del Reino Unido con COVID-19 utilizando el Protocolo de caracterización clínica ISARIC de la OMS. medRxiv [Internet]. 2020 [Consultado 2021 May 01] Disponible en: https://doi.org/10.1101/2020.04.23.20076042

23. Richardson S, Hirsch JS, Narasimhan M, Crawford JM, McGinn T, Davidson KW. Presenting Characteristics, Comorbidities, and Outcomes among 5700 Patients Hospitalized with COVID-19 in the New York City Area. JAMA - Journal of the American Medical Association. 2020; 323: 2052-9.
24. Simonnet A, Chetboun M, Poissy J, Raverdy V, Noulette J, Duhamel A. High Prevalence of Obesity in Severe Acute Respiratory Syndrome Coronavirus-2 (SARS-CoV-2) Requiring Invasive Mechanical Ventilation. Obesity. 2020; 28(7): 1195-9.

25. Caussy C,Wallet F,Laville M, Disse E. Obesity is Associated With Severe Forms os COVID-19. Obesity. 2020; 28(7): 1175.

26. Alvárez Ochoa RI, Gualpa Lema MC, Sacoto Naspud NT, Guallpa González MJ, Cordero Cordero Gd. Factores de riesgo cardiovascular en profesionales de enfermería. Rev cuba med gen integr [Internet]. 2018 [Consultado 2021 May 22]; 34(2). Disponible en: http://www.revmgi. sld.cu/index.php/mgi/article/view/468

27. Codas M, Chamorro L, Figueredo M, Achucarro D,Martínez $V$, Estilos de vida y riesgo cardiovascular y cardiometabólico en profesionales de salud del Hospital Regional de Encarnación. Rev. Virtual Soc. Parag. Med. Int. 2018; 5(1): 12-24.

28. Fernández Martín G, López Gallego M, Delgado Lozano $C$. Juicio de las enfermeras acerca de un plan de cuidados estandarizado de infección por coronavirus (COVID-19). Revista Enfermería Docente. 2021; Primer Semestre 2021(113): 33-38.

29. Herdman TH, Kamitsuru S. Diagnósticos Enfermeros. Barcelona: NANDA Internacional; 2018-2020.

30. Moor S,Moohead MJ, Meridean L. Maas ES. Clasificación de resultados de Enfermería (NOC) Medición de Resultados en Salud Sue Moorhead. $5^{\mathrm{a}}$ ed. España: Elsevier; 2013.

31. Das S, Anu KR, Birangal SR, Nikam AN, Pandey A, Mutalik $\mathrm{S}$, Joseph A. Papel de las comorbilidades como la diabetes en el síndrome respiratorio agudo severo coronavirus-2: una revisión. Ciencias de la vida, 258(2020): 6 Disponible en: https://doi.org/10.1016/j.Ifs.2020.118202 